\title{
The Hirota Method for Reaction-Diffusion Equations with Three Distinct Roots
}

\author{
Gamze Tanoğlu* and Oktay Pashaev* \\ * Department of Mathematics, Izmir Institute of Technology, \\ Gulbahce Campus, 35430, Urla, Izmir, Turkey
}

\begin{abstract}
The Hirota Method, with modified background is applied to construct exact analytical solutions of nonlinear reaction-diffusion equations of two types. The first equation has only nonlinear reaction part, while the second one has in addition the nonlinear transport term. For both cases, the reaction part has the form of the third order polynomial with three distinct roots. We found analytic one-soliton solutions and the relationships between three simple roots and the wave speed of the soliton. For the first case, if one of the roots is the mean value of other two roots, the soliton is static. We show that the restriction on three distinct roots to obtain moving soliton is removed in the second case by, adding nonlinear transport term to the first equation.
\end{abstract}

Key words. Soliton, Hirota method, diffusion- reaction equations, phase transition.

PACS. 02.30.jr, 05.45.-a, 11.10.Lm.

MSC. 35Q51, 35K57.

\section{INTRODUCTION}

There is a big variety of nonlinear phenomena which can be well described by travelling waves. They include, genetic waves in biology, vibrations in physics, phase transitions and interface phenomena in material science, and so on [1]. In the systems with degenerate ground state the kink type travelling wave implements transition between two ground state configurations. The speed of such wave is an important characteristic related with ground state values of the order parameter. According to the Landau theory of the phase transitions, the potential in effective energy has form of polynomial function of the order parameter. The simplest potential admitting two ground states and the kink soliton between these states has to be polynomial degree of four. Then, the effective force in dynamical equation for the order parameter is of the cubic form. According to the main theorem of algebra, cubic polynomial with real coefficients admits only one or three real roots. But kink type travelling wave for the real order parameter could exist only for effective force with three distinct roots.

In the present paper we consider non-linear diffusion equation,

$$
U_{t}=U_{x x}-\left(U-a_{1}\right)\left(U-a_{2}\right)\left(U-a_{3}\right),
$$

with nonlinear reaction term having three distinct roots $a_{1}, a_{2}, a_{3}$. Frequently it is called as Fisher's type equation. There are many papers dealing with solutions of this equation 
for the degenerate roots and with different applications of it. The main progress has been made to find travelling wave solution of the reaction-diffusion equations, by travelling wave ansatz, reducing a nonlinear PDE to a nonlinear ODE. But in ODE system, the travelling wave speed is an unknown parameter that must be fixed by the analysis and choosing special trial trajectory [1]. That is why the method seems doesn't allow extensions to more than one travelling waves, higher space dimensions and multi-component order parameter. From another site, during the last 30 years, the direct method proposed by Hirota has become a powerful tool to construct multi-soliton solutions of integrable systems [3]. This, relatively simple and algebraic rather than analytic method, allows one to avoid many analytic difficulties of more sophisticated the inverse scattering method. Moreover, it is deeply related with Plucker coordinates of Grassmanians, quantum theory of fermions, $\tau$ functions and vertex operator representation of infinite-dimensional algebras [2]. Recently, the Hirota method has been applied also to construct exact solution of some non-integrable evolution equations. The general idea of the method is first to transform nonlinear equation under consideration into bilinear equation, and then use the power series expansion to solve it. For integrable systems the series admits exact truncation for arbitrary number of solitons. While for periodic solutions it includes an infinite number of terms. To find proper bilinearization in the case of Eq. (1) with cubic nonlinearity, we propose to extend the Hirota ansatz by extracting one of the roots as a background field. Then, solution of bilinear equations gives travelling wave, implementing transition between other two roots and with velocity, depending on values of all three roots. Efficiency and simplicity of our approach is demonstrated by exact integration of extended model with additional nonlinear transport term,

$$
U_{t}+\alpha U U_{x}=U_{x x}-\left(U-a_{1}\right)\left(U-a_{2}\right)\left(U-a_{3}\right),
$$

where $\alpha$ is a constant.

The paper is organized as follows. In the next section, modified Hirota Ansatz is applied to find one soliton solution for the equation (1). Firstly, Eq. (1) is transformed into the bilinear system of differential equations, which we solve exactly using a kind of perturbational approach. We show that if the background root of our ansatz is the mean value of the other two roots, the velocity parameter is vanishing and soliton is static. In the following section, by adding the nonlinear transport term to Eq. (1) we study Eq. (2). The related, coupled Hirota bilinear form is obtained and the one soliton solution is found. We show that even in the mean value case our solution is not static. To be the case, parameter $\alpha$ must be fixed as some definite function of roots $a_{1}, a_{2}, a_{3}$. In conclusions we discuss some applications and possible extensions of our results to other equations.

\section{REACTION- DIFFUSION EQUATION}

To reduce Eq. (1) with cubic nonlinearity and three distinct roots to the bilinear form, we have to modify the standard Hirota ansatz. The solution of the problem is assumed to have a form

$$
U=a_{i}+\frac{g}{f}
$$


where $\mathrm{i}=1,2,3$ and $g, f$ are real functions of $x$, $t$. For three different numbers, $a_{1}, a_{2}, a_{3}$ it provides three different solutions of the same form, but with different parameters. Thus, without loss of generality we assume that $i=3$.

In order to obtain bilinear system of equations, all derivatives with respect to the dependent variables in Eq. (1) are expressed as

$$
\begin{gathered}
U_{t}=\frac{D_{t}(g \cdot f)}{f^{2}}, U_{x}=\frac{D_{x}(g \cdot f)}{f^{2}}, \\
U_{x x}=\frac{D_{x}^{2}(g \cdot f)}{f^{2}}-\frac{g}{f} \frac{D_{x}^{2}(f \cdot f)}{f^{2}},
\end{gathered}
$$

where the Hirota derivative operator is defined as

$$
D_{x}^{n}(a \cdot b)=\left.\left(\frac{\partial}{\partial x_{1}}-\frac{\partial}{\partial x_{2}}\right)^{n}\left(a\left(x_{1}\right) b\left(x_{2}\right)\right)\right|_{x=x_{1}=x_{2}} .
$$

After substituting Eqs. (4) into Eq. (1), the following expression is obtained:

$$
\frac{D_{t}(g \cdot f)}{f^{2}}-\frac{D_{x}^{2}(g \cdot f)}{f^{2}}+\frac{g}{f}\left[\frac{D_{x}^{2}(f \cdot f)}{f^{2}}+\left(\frac{g}{f}-\left(a_{1}-a_{3}\right)\right)\left(\frac{g}{f}-\left(a_{2}-a_{3}\right)\right)\right]=0
$$

Since in Eq.(3) instead of one real function $U$ we introduced two real functions $g$ and $f$, we have freedom to decouple this equation on two bilinear ones. Equating part in brackets to zero, we deduce the following bilinear system

$$
\begin{array}{r}
\left(D_{t}-D_{x}^{2}\right)(g \cdot f)=0, \\
\left(D_{x}^{2}+\tilde{a_{1}} \tilde{a_{2}}\right)(f \cdot f)=-g^{2}+\left(\tilde{a_{1}}+\tilde{a_{2}}\right) g f,
\end{array}
$$

where $\tilde{a_{1}} \equiv a_{1}-a_{3}$ and $\tilde{a_{2}} \equiv a_{2}-a_{3}$. To solve this system in the Hirota method functions $\mathrm{f}$ and $\mathrm{g}$ suppose to have the form of the perturbation series in some parameter $\varepsilon$

$$
\begin{gathered}
f=1+\varepsilon f_{1}+\varepsilon^{2} f_{2}+\ldots . ., \\
g=g_{0}+\varepsilon g_{1}+\varepsilon^{2} \varepsilon g_{2}+\ldots . .
\end{gathered}
$$

Substituting (10) into the system (8) and equating coefficients of the same power of $\varepsilon$, converts (8) into a sequence of bilinear equations of the zeroth, first, second and so on orders respectively:

$$
\begin{gathered}
\left(D_{t}-D_{x}^{2}\right)\left(g_{0} \cdot 1\right)=0, \\
\left(D_{x}^{2}+\tilde{a_{1}} \tilde{a_{2}}\right)(1 \cdot 1)=-g_{0}^{2}+\left(\tilde{a_{1}}+\tilde{a_{2}}\right) g_{0} ; \\
\left(D_{t}-D_{x}^{2}\right)\left(g_{0} \cdot f_{1}+g_{1} \cdot 1\right)=0, \\
\left(D_{x}^{2}+\tilde{a_{1}} \tilde{a_{2}}\right)\left(2 \cdot f_{1}\right)=-\left[2 g_{0} g_{1}-\left(\tilde{a_{1}}+\tilde{a_{2}}\right)\left(g_{0} f_{1}+g_{1} \cdot 1\right)\right] ; \\
\left(D_{t}-D_{x}^{2}\right)\left(g_{0} \cdot f_{2}+g_{1} \cdot f_{1}+g_{2} \cdot 1\right)=0, \\
\left(D_{x}^{2}+\tilde{a_{1}} \tilde{a_{2}}\right)\left(2 \cdot f_{2}+f_{1} \cdot f_{1}\right)=-2 g_{0} g_{2}-g_{1}^{2}+\left(\tilde{a_{1}}+\tilde{a_{2}}\right)\left(g_{0} f_{2}+g_{1} f_{1}+g_{2} \cdot 1\right) ;
\end{gathered}
$$




\subsection{The zeroth order solution}

We assume that $g_{0}$ is a constant, then the first equation in (12) is satisfied automatically. From the second equation (12), we get

$$
g_{0}^{2}-\left(\tilde{a_{1}}+\tilde{a_{2}}\right) g_{0}+\tilde{a_{1}} \tilde{a_{2}}=\left(g_{0}-\tilde{a_{1}}\right)\left(g_{0}-\tilde{a_{2}}\right)=0,
$$

which is the quadratic algebraic equation for $g_{0}$ with two roots $\tilde{a_{1}}$ and $\tilde{a_{2}}$.

\subsection{The first order solution}

In this subsection we find the first order solutions, $g_{1}, f_{1}$. First, without loss of generality we assume that $g_{0}=\tilde{a_{1}}$. Then Eqs. (14) may be rewritten as a linear system

$$
\begin{array}{r}
\tilde{a_{1}}\left(-\partial_{t}-\partial_{x}^{2}\right) f_{1}+\left(\partial_{t}-\partial_{x}^{2}\right) g_{1}=0, \\
2 f_{1_{x x}}+2 \tilde{a_{1}} \tilde{a_{2}} f_{1}+2 \tilde{a_{1}} g_{1}-\left(\tilde{a_{1}}+\tilde{a_{2}}\right)\left(\tilde{a_{1}} f_{1}+g_{1}\right)=0 .
\end{array}
$$

The simplest nontrivial solution of this system has the form

$$
g_{1}=e^{\eta_{1}}, f_{1}=b e^{\eta_{1}}
$$

where $\eta_{1}=k_{1} x+\omega_{1} t+\gamma_{1}$. Coefficient in front of $g_{1}$ can be absorbed by constant $\gamma_{1}$, it is why we can choose it equal to one. Unknown constants $k_{1}$ and $\omega_{1}$ are fixed by dispersion relation. After substituting Eq. (22) into the system (20) we have the algebraic system. The nontrivial solution of this system exists only if the following dispersion relation on $k_{1}$ and $\omega_{1}$ is satisfied

$$
\omega_{1}=k_{1}^{2}+\tilde{a_{1}}\left(\tilde{a_{2}}-\tilde{a_{1}}\right) .
$$

The free parameter $b$ becomes fixed as

$$
b=\frac{\left(\tilde{a_{2}}-\tilde{a_{1}}\right)}{2 k_{1}^{2}+\tilde{a_{1}}\left(\tilde{a_{2}}-\tilde{a_{1}}\right)} .
$$

So, from above the calculations only $k_{1}$ and $\gamma_{1}$ are free parameters of our solution.

\subsection{The second order calculation}

Substituting $g_{1}, f_{1}$ to the system (16) and using property of bilinear Hirota operators

$$
\left(D_{t}-D_{x}^{2}\right)\left(g_{1} \cdot f_{1}\right)=b\left(D_{t}-D_{x}^{2}\right)\left(e^{\eta_{1}} \cdot e^{\eta_{1}}\right)=0
$$

for $g_{2}, f_{2}$ we find the following equation

$$
\tilde{a_{1}}\left(-\partial_{t}-\partial_{x}^{2}\right) f_{2}+\left(\partial_{t}-\partial_{x}^{2}\right) g_{2}=0
$$


similar to Eq.(20). The simplest solutions for this equation is the trivial one $g_{2}=0$ and $f_{2}=0$. Then from Eq.(16) we find additional constraint on $g_{1}, f_{1}$ and $b$ :

$$
\tilde{a_{1}} \tilde{a_{2}} f_{1}^{2}=-g_{1}^{2}+\left(\tilde{a_{1}}+\tilde{a_{2}}\right)\left(g_{1} f_{1}\right)
$$

Substitution of Eq. (22) into Eq. (27)results in quadratic equation for $b$ :

$$
\tilde{a_{1}} \tilde{a_{2}} b^{2}-\left(\tilde{a_{1}}+\tilde{a_{2}}\right) b+1=\left(\tilde{a_{1}} b-1\right)\left(\tilde{a_{2}} b-1\right)=0
$$

The roots of this quadratic equation are

$$
b_{1}=\frac{1}{\tilde{a_{1}}}, b_{2}=\frac{1}{\tilde{a_{2}}} .
$$

If we assume that $b=\frac{1}{\tilde{a}_{1}}$, then after simple calculation we find that the solution of our problem becomes constant, i.e, $u=a_{1}$. Therefore, we assume that $b=\frac{1}{\tilde{a}_{2}}$. Combining this condition with Eq.(24), we find restrictions on allowed values of parameter $k_{1}$ by Eq.(31). It is easy to show that each bilinear equation, which has order greater than 2 , has simple solution as $g_{i}=0$ and $f_{i}=0$ for $i>2$. Therefore, we have only finite number of terms. After substituting $f$ and $g$ into the form of $u$, we obtain the exact solution of our problem as follows :

$$
u=a_{3}+\frac{\left(a_{1}-a_{3}\right)+e^{\eta_{1}}}{\left(a_{2}-a_{3}\right)+e^{\eta_{1}}}\left(a_{2}-a_{3}\right) .
$$

This is the kink-soliton with asymptotics $u \rightarrow a_{1}$, for $x \rightarrow-\infty$ and $u \rightarrow a_{2}$, for $x \rightarrow+\infty$, at fixed time.

If in the zeroth order calculations we choose instead of $\tilde{a}_{1}$ the second root $g_{0}=\tilde{a}_{2}$ and in the second order calculations (29), $b=1 / \tilde{a}_{1}$, then we get another solution with opposite asymptotics $u \rightarrow a_{2}$, for $x \rightarrow-\infty$ and $u \rightarrow a_{1}$, for $x \rightarrow+\infty$.

\subsection{Velocity of the soliton}

In this section we find the wave number parameter $k_{1}$, frequency $\omega_{1}$ and the speed of the soliton $\mathrm{v}$ in terms of three distinct roots, $a_{1}, a_{2}, a_{3}$. By equating Eqs. (24) and (29), we get

$$
k_{1}= \pm \frac{1}{\sqrt{2}}\left(a_{2}-a_{1}\right)
$$

and from Eq.(23)

$$
\omega_{1}=\frac{1}{2}\left(a_{2}^{2}-a_{1}^{2}\right)-a_{3}\left(a_{2}-a_{1}\right) .
$$

The velocity $\mathrm{v}$ of our travelling wave is given by the equation

$$
\mathrm{v}=\left(-\frac{\omega_{1}}{k_{1}}\right)
$$


or combining Eqs. (31) and (32) we obtain the following relationship between the velocity and the roots of the nonlinear part.

$$
\mathrm{v}= \pm \frac{a_{1}+a_{2}-2 a_{3}}{\sqrt{2}} .
$$

When the root $a_{3}$ is equal to the mean value of two other roots $a_{3}=\left(a_{1}+a_{2}\right) / 2$, the velocity vanishes and soliton is static. By cyclic permutation of indices 1,2,3 in Eqs.(3) and (30) we find other kink solitons of our problem. But only one of them could be stable since implement transition between stable vacuum states.

\section{EXTENDED REACTION-DIFFUSION EQUATION}

In this section, we solve Eq. (2) by modified Hirota method introduced in section 1. We use the same ansatz for $U$ and perturbation series expansion of $f$ and $g$. After using Hirota derivatives (4), the analog form to the Equation (7) can be obtained for the Eq. (2) as well, then decoupling this equation, the following bilinear system of Eqs. can be deduced

$$
\begin{array}{r}
\left(D_{t}+\alpha a_{3} D_{x}-D_{x}^{2}\right)(g \cdot f)=0, \\
\left(D_{x}^{2}+\tilde{a_{1}} \tilde{a_{2}}\right)(f \cdot f)=-g^{2}+\left(\tilde{a_{1}}+\tilde{a_{2}}-\alpha D_{x}\right)(g f) .
\end{array}
$$

After substituting the previous ansatz for $f$ and $g$, we obtain the zeroth, first and second order bilinear equations. The zeroth order solution will not change. For the first order solution, we again assume that the form of the $f$ and $g$ are the same as in Eq. (22). After substituting ( 22) into the Equations (35), we obtain the algebraic system which has nontrivial solution if and only if the following dispersion relation on parameters $k_{1}$, $\omega_{1}$ and $\alpha$ is satisfied

$$
\omega_{1}=k_{1}^{2}+\tilde{a_{1}}\left(\tilde{a_{2}}-\tilde{a_{1}}\right)-\left(\tilde{a_{1}}+a_{3}\right) \alpha k_{1} .
$$

Then, parameter $b$ becomes fixed as

$$
b=\frac{\left(\tilde{a_{2}}-\tilde{a_{1}}\right)-\alpha k_{1}}{2 k_{1}^{2}+\tilde{a_{1}}\left(\tilde{a_{2}}-\tilde{a_{1}}\right)-\tilde{a_{1}} \alpha k_{1}} .
$$

If we put $\alpha=0$ in the Eqs. (37) and (38), one can see easily show that the equations reduce to Eqs. (23) and (24), respectively.

The second order calculation will not change, and one can find that value of $b$ is as before. Then, by equating the Eqs. (29) and (38), we find following restriction on $k_{1}$,

$$
k_{1}=\frac{\left(\tilde{a}_{2}-\tilde{a}_{1}\right)\left(-\alpha \mp \varepsilon \sqrt{\alpha^{2}+8}\right)}{4},
$$

where

$$
\varepsilon=\left\{\begin{array}{cc}
1, & a_{2}>a_{1} \\
-1, & a_{2}<a_{1} .
\end{array}\right.
$$


Finally, the velocity of our solution can be calculated by using Eq. (33), where $\omega_{1}$ and $k_{1}$ are given by Eqs. (37) and (39), respectively:

$$
\mathrm{v}=\frac{2\left(a_{1}+a_{2}-2 a_{3}\right)}{\beta}-\left(\frac{a_{2}+a_{1}}{2}\right) \alpha,
$$

where $\beta=-\alpha \pm \varepsilon \sqrt{\alpha^{2}+8}$. From the last equation one can see that even for the mean value condition $a_{3}=\left(a_{1}+a_{2}\right) / 2$ the soliton is not static. The static soliton arises for fixed value of $\alpha$ in terms of $a_{1}, a_{2}, a_{3}$ in Eq.(40).

\section{CONCLUSION}

In the present paper, the modified Hirota method is applied to find the exact analytic one soliton solution of the nonlinear reaction-diffusion equations. These equations have nonlinear reaction part which is the third order polynomial with three distinct roots. In the phase transition content, these three distinct roots correspond to the order of the system phases. From the phase plane analysis, the system has two stable and one unstable phases or one stable and two unstable phases. In the last case no stable kink soliton can exist. The solutions of equations (1) and (2) giving connection between two stable phases correspond to the one soliton solutions. If solution of the problem is written with background value of the one of the unstable phases, say $a_{3}$, then it gives connection between two stable phases, $a_{1}$ and $a_{2}$.

Finally, we showed that Hirota method is very efficient and systematic procedure to obtain exact solutions of reaction-diffusion equations with three distinct roots. We hope that the method allows one to find solutions of other extensions of the model to higher dimensions, additional terms and multi-component order parameters. These questions are under our study now.

\section{ACKNOWLEDGMENTS}

This work was supported partially by Izmir Institute of Technology grant 2002-IYTE24 and 2002-IYTE-25.

\section{REFERENCES}

1. Alwyn Scott, Nonlinear Science, Oxford University Press, 1999

2. Alan C. Newell, Soliton and Physics, SIAM, 1985.

3. R. Hirota, Direct methods in Soliton Theory, in : "Solitons, Springer Verlag", 1980. 